Algebraic 83 Geometric $\mathcal{T}$ opology

Volume 5 (2005) 751-768

Published: 23 July 2005

ATG

\title{
Bootstrapping in convergence groups
}

\author{
ERIC L. SWENSON
}

\begin{abstract}
We prove a true bootstrapping result for convergence groups acting on a Peano continuum.

We give an example of a Kleinian group $H$ which is the amalgamation of two closed hyperbolic surface groups along a simple closed curve. The limit set $\Lambda H$ is the closure of a "tree of circles" (adjacent circles meeting in pairs of points). We alter the action of $H$ on its limit set such that $H$ no longer acts as a convergence group, but the stabilizers of the circles remain unchanged, as does the action of a circle stabilizer on said circle. This is done by first separating the circles and then gluing them together backwards.
\end{abstract}

AMS Classification 20F32; 57N10

Keywords Convergence group, bootstrapping, Peano continuum

\section{Introduction}

When a group $G$ acts by homeomorphisms on a compact metric space $X$, then, in general, little can be said about $G$ or $X$. However if $G$ acts on $X$ as a convergence group then we can often say much more. For instance if $X$ is a topological circle then $G$ "is" a Fuchsian group where the action of $G$ on $X$ is given by the Fuchsian action on the circle at infinity of $G$ [14, 10, 8]. For any metric space, if $G$ acts on $X$ as a uniform convergence group, then $G$ is word hyperbolic and $X=\partial G$ [2]. More generally if $G$ acts on $X$ as a geometrically finite convergence group, then $G$ is relatively hyperbolic with boundary $X[16$.

The purpose of this note is to examine what conditions will allow us to prove that $G$ acts on $X$ as a convergence group. In particular we are interested in "bootstrapping", that is using subgroups (with presumably smaller limit sets) which act as convergence groups to prove that $G$ acts as a convergence group on $X$. We will restrict ourselves to that case where $X$ is a Peano continuum without cut points. This restriction is justified to some extent by the results of [1, [3, 4] and [5]. This is a continuation of a program began in [12], 13]. 
Our goal (which we achieve) is a natural bootstrapping result when $X$ is an $n$-sphere $(n>1)$ and the subgroups have limit sets $(n-1)$-spheres in $X$. This follows from our main theorem. (Recall that a map is essential if it is not homotopic to a constant map)

Main Theorem Let $X$ be a Peano continuum, without cut points, which does not admit an essential map to the circle. If $G$ acts on $X$ by homeomorphisms and $\mathcal{A}$ is a $G$-invariant collection of connected closed subsets of $X$, then $G$ acts as a convergence group on $X$ provided the following conditions are satisfied:

- $\mathcal{A}$ is null, that is for any $\epsilon>0$, the set of elements of $\mathcal{A}$ with diameter at least $\epsilon,\{A \in \mathcal{A}: \operatorname{diam}(A)>\epsilon\}$, is finite.

- $\mathcal{A}$ is fine, that is for any $x, y \in X$ there exists a finite $\mathcal{B} \subset \mathcal{A}$ such that $\cup \mathcal{B}$ separates $x$ from $y$.

- $\operatorname{Stab}(A)$ acts as a convergence group on $A$ for each $A \in \mathcal{A}$.

We also have results which apply when the elements of $\mathcal{A}$ are not connected, but they are more technical.

\section{Background}

Definition Let $G$ be a group acting by homeomorphisms on a space $X$, a sequence $\left(g_{i}\right) \subset G$ acts as a convergence sequence on $X$ if $\exists n, p \in X$ such that for any compact $C \not \supset n, g_{i}(C) \rightarrow p$. We call $n$ the repeller and $p$ the attractor of $\left(g_{i}\right)$ in its action on $X$. (It is possible that $n=p$ ). It is easily shown that if $\left(g_{i}\right)$ acts as a convergence sequence on $X$ with attractor $p$ and repeller $n$, then $\left(g_{i}^{-1}\right)$ acts as a convergence sequence on $X$ with attractor $n$ and repeller $p$. We say that $G$ acts as a convergence group on $X$ if every sequence of distinct elements of $G$ has a subsequence acting as a convergence sequence on $X$.

A continuum is a compact connected metric space, and a Peano continuum is a locally connected continuum. For $A \subset X$ and $B, C$ connected subsets of $X-A$ we say $A$ separates $B$ from $C$ if $B$ and $C$ lie in different components of $X-A$. We say that $A$ separates $Y \subset X$ if $A$ separates two points of $Y$.

Definition Let $A$ and $B$ be closed sets of a Peano Continuum $X$. We say $A$ and $B$ cross if either $A \cap B \neq \emptyset$ or $A$ separates $B$ and $B$ separates $A$.

A sequence $A_{1}, \ldots A_{n}$ is called a crossing sequence from $A_{1}$ to $A_{n}$ if $A_{i}$ crosses $A_{i+1}$ for all $1 \leq i<n$. The sequence is called a minimal crossing sequence if $A_{i}$ doesn't cross $A_{j}$ for $|i-j|>1$. 
Definition Let $X$ be a Peano continuum, and $G$ be a group which acts by homeomorphisms on $X$. If $\mathcal{A}$ is a $G$-invariant collection of closed subsets of $X$, we say that the pair $(G, \mathcal{A})$ is a fine pairing on $X$ if the following conditions are satisfied:

(1) $\mathcal{A}$ is cross connected, that is for any $A, B \in \mathcal{A}$ there is a crossing sequence in $\mathcal{A}$ from $A$ to $B$.

(2) $\mathcal{A}$ is null. That is: For any $\epsilon>0$, the set of elements of $\mathcal{A}$ with diameter at least $\epsilon,\{A \in \mathcal{A}: \operatorname{diam}(A)>\epsilon\}$ is finite.

(3) $\mathcal{A}$ is fine. That is: For any $x, y \in X$ there exists a finite $\mathcal{B} \subset \mathcal{A}$ such that $\cup \mathcal{B}$ separates $x$ from $y$.

The following is the main result of [13].

Theorem 1 Let $X$ be a Peano continuum without cut points, and $(G, \mathcal{A})$ be a fine pairing on $X$. If, for each $A \in \mathcal{A}, \operatorname{Stab}(A)$ acts as a convergence group on $X$, then $G$ acts as a convergence group on $X$.

The difficulty with applying this result is that in order to prove that $G$ acts as a convergence group on $X$ we must prove that each element of an infinite family of subgroups acts as a convergence group on $X$. If, in the above result, we could replace the phrase " $\operatorname{Stab}(A)$ acts as a convergence group on $X$ " with the phrase "Stab $(A)$ acts as a convergence group on $A$ ", then we would be able to bootstrap. This was what the author set out to prove. The following counterexample explains why he failed.

\section{Counterexample}

The idea of this counterexample is quite simple, but the details are not. What we will do is start with a Kleinian group $H$ whose limit set is a "tree" of circles in $S^{2}$ (where a pair of circles which intersect one another do so in a pair of points which are the endpoints of a hyperbolic element). In fact $H$ will be the free product of two surface groups amalgamated along an element corresponding to a simple closed curve in both. We now take the tree of circles, pull out every other circle in it and glue them back in interchanging each pair of points in an intersection. The action of $H$ on $\Lambda H$ will result in an action on the new tree of circles, but the action of $H$ on this new tree of circles will not be a convergence action even though it satisfies all of the conditions of the conjecture that the author wished to prove. 
This example is constructed using only what the author considers to be the most elementary of techniques at his disposal. An expert in Kleinian groups could complete this construction much faster, and without any need for the geometric group theory arguments which the author employs.

Let $F$ be the Kleinian group generated by reflection in the faces of a regular right-angled hyperbolic dodecahedron $D$. Let $P$ (purple) and $Y$ (yellow) be hyperbolic planes which contain adjacent faces (that is to say faces which intersect in an edge) of $D$. Let $F_{P}=\operatorname{Stab}(P)$, the stabilizer of $P$ in $F$, and similarly define $F_{Y}=\operatorname{Stab}(Y)$. Clearly $P / F_{P}$ is a (purple) closed hyperbolic orbifold and similarly $Y / F_{Y}$ is a (yellow) closed hyperbolic orbifold. The intersection line $P \cap Y$ descends to a closed geodesic in both $P / F_{P}$ and $Y / F_{Y}$, in fact both $P / F_{P}$ and $Y / F_{Y}$ are regular right angled hyperbolic pentagon reflection orbifolds, and the intersection line will descend to an edge of the orbifold. Notice that there is an isomorphism $\chi: P / F_{P} \rightarrow Y / F_{Y}$ of these orbifolds which sends the intersection edge in $P / F_{P}$ to the intersection edge in $Y / F_{Y}$.

Fix $d>0$. Using classical Fuchsian group results we can find a finite index subgroup $H_{P}<F_{P}$, so that letting $H_{Y}=\chi\left(H_{P}\right)<F_{Y}$ we have:

(1) The quotients $P / H_{P}$ and $Y / H_{Y}$ are orientable closed surfaces.

(2) The intersection line $P \cap Y$ descends to the simple closed intersection curve in each of $P / H_{P}$ and $Y / H_{Y}$.

(3) $H_{P} \cap H_{Y}=\langle g\rangle$ where $\langle g\rangle$ is the stabilizer of $P \cap Y$ in both $H_{P}$ and $H_{Y}$.

(4) Every closed curve on $P / H_{P}$ or $Y / H_{Y}$ which intersects the intersection curve transversely has length at least $d$.

Let $H=\left\langle H_{P}, H_{Y}\right\rangle$, the subgroup of $F$ generated by $H_{P}$, and $H_{Y}$.

We will show that for $d \gg 0, H=H_{P} *\langle g\rangle H_{Y}$, the free product of $H_{P}$ with $H_{Y}$ amalgamated over $\langle g\rangle$. Let $\mathcal{P}=\{h(P) \mid h \in H\}$ be the purple planes (translates of $P$ by $H$ ) and $\mathcal{Y}=\{h(Y) \mid h \in H\}$ be the yellow planes (translates of $Y$ ).

We show that $P$ is not a translate of $Y$ in $H$ by showing that $P$ is not a translate of $Y$ in our original reflection group $F$. To do this give each face of $D$ its own color, and tile $\mathbb{H}^{3}$ with copies of $D$ by reflecting in the faces of $D$. Notice that after reflection in a single face of $D$, the resulting 17-hedron (2 of the pentagon faces will be in the interior of the solid, and 10 pentagons will be amalgamated into 5 hexagon faces) will have each face of one color. It follows that each plane in the tiling of $\mathbb{H}^{3}$ will have only one color (each plane will be tiled with pentagons of the same color). Thus $P$ is not a translate of $Y$ in $F$ or in $H$, so $\mathcal{P} \cap \mathcal{Y}=\emptyset$. 
We construct a bipartite graph $T$ with alternating purple and yellow vertices in the obvious way. Let $T^{(0)}=\mathcal{P} \cup \mathcal{Y}$ so the vertices are the purple and yellow planes, and we connect two vertices with an edge if and only if the corresponding planes intersect. The group $H$ acts on $T$ with out fixed points.

Definition Let $x_{0}, x_{1}, \ldots x_{m} \in \mathbb{H}^{3}$. The union of the geodesic segments $\alpha=$ $\left[x_{0}, x_{1}\right] \cup\left[x_{1}, x_{2}\right] \cup\left[x_{2}, x_{3}\right] \cdots \cup\left[x_{n-1}, x_{n}\right]$ is called a broken geodesic from $x_{0}$ to $x_{n}$, with break points $x_{1}, \ldots x_{n-1}$. The length $\ell(\alpha)=\sum \ell\left(\left[x_{i-1}, x_{i}\right]\right)$ is the sum of the lengths of the segments. The angle between the segments $\left[x_{i-1}, x_{i}\right]$ and $\left[x_{i}, x_{i+1}\right]$ is called the break angle of $\alpha$ at $x_{i}$.

In [6], Cannon proved the following theorem.

Theorem 2 For any $\theta>0$ there exists $d(\theta)>0$ and $\epsilon(\theta)>0$ with the following property: For any broken geodesic $\alpha=\bigcup_{i=1}^{n}\left[x_{i-1}, x_{i}\right]$, with break angles at least $\theta$, satisfying $\ell\left(\left[x_{i-1}, x_{i}\right]\right) \geq d(\theta)$ for all $1<i<n$ and for any $a, b \in \alpha$, then $d(a, b) \geq \epsilon(\theta) \ell\left(\alpha^{\prime}\right)$ where $\alpha^{\prime}$ is the subpath of $\alpha$ from $a$ to $b$.

When $d \geq d\left(\frac{\pi}{2}\right)$, using Cannon's result we show that $T$ is a tree. Suppose not. There is a minimal circuit in $T$, and from this we construct a broken geodesic closed curve $\alpha \subset \cup(\mathcal{P} \cup \mathcal{Y}) \subset \mathbb{H}^{3}$ where each geodesic segment in $\alpha$ is $\alpha \cap B$ where $B$ is one of the vertex planes of the circuit. Homotopying $\alpha$ relative to the intersection lines of our vertex planes, we may assume that $\alpha$ has minimal length, that is to say no homotopy of $\alpha$ relative to the intersection lines has shorter length. (This argument is made very easy by the fact that the intersection lines diverge from one another exponentially).

Notice that every segment of $\alpha$ has length at least $d$ by (4). We show that no break angle in $\alpha$ is acute. Consider a break point $q \in \alpha$, lying on the intersection of planes $P^{\prime} \in \mathcal{P}, Y^{\prime} \in \mathcal{Y}$. Since $P^{\prime} \perp Y^{\prime}$, there is a unique hyperbolic plane $Q \ni q$ with $Q \perp P^{\prime}$ and $Q \perp Y^{\prime}$.

If the interiors of the 2 segments of $\alpha$ which meet at $q$ lie on same side of $Q$ (aside from the endpoint $q$ ) then we can shorten $\alpha$ by sliding $q$ to that side of $Q$, and if not then the break angle is not acute. Thus the break angle of $\alpha$ at $q$ is not acute.

Every segment is at least $d$ long, so by Cannon's theorem $d(q, q) \geq \epsilon\left(\frac{\pi}{2}\right) \ell(\alpha)>$ 0 . This is a contradiction, so $T$ is a tree. A ping pong argument now shows that the stabilizer of $P$ in $H$ is $H_{P}$ and similarly the stabilizer of $Y$ in $H$ is $H_{Y}$. Thus $H=H_{P} *\langle g\rangle H_{Y}$ 
Consider the Poncaire ball model for $\mathbb{H}^{3}$, the ball of radius 1 about $\mathbf{0}$. Position $D$ so that $\mathbf{0} \in P \cap Y$. We move our perspective to the boundary $S^{2}=\{\mathbf{x} \in$ $\left.\mathbb{R}^{3} \mid\|\mathbf{x}\|=1\right\}$ of the ball model for $\mathbb{H}^{3}$. Each hyperbolic plane in $\mathbb{H}^{3}$ corresponds to a circle in $S^{2}$. Thus the purple planes correspond to purple circles in $S^{2}$ and similarly the yellow planes correspond to yellow circles. We will denote by $\pi$ the boundary circle of $P$, and by $v$ the boundary circle of $Y$. Two planes intersect in a line if and only if the corresponding circles intersect in two points. Let $\Pi$ be the collection of all the purple circles $(\Pi=\{\partial \breve{P} \mid \breve{P} \in \mathcal{P}\})$, and similarly let $\Upsilon$ be the collection of yellow circles $(\Upsilon=\{\partial \breve{Y} \mid \breve{Y} \in \mathcal{Y}\})$.

We now redefine the vertex set of $T$ to be $\Pi \cup \Upsilon$ and two vertices are connected by an edge if the vertex circles intersect in two points. Of course $T$ is still (the same) bipartite (yellow and purple) tree.

Let

$$
X=\cup(\Pi \cup \Upsilon) \subsetneq \Lambda H \subset S^{2} .
$$

As we will see, $X$ is a finite diameter path metric space. For this we need the following result of negative curvature due to Gromov [11, Cannon [6], 7] and others.

Proposition 3 For any $\epsilon>0$ there is a $\delta>0$ such that if $p:[a, b] \rightarrow$ $\mathbb{H}^{n}$ is a path satisfying $d(p(x), p(y)) \geq \epsilon \ell(p([x, y]))$ then $p$ and the geodesic from $p(a)$ to $p(b)$ fellow travel, $[p(a), p(b)] \subset \operatorname{Nbh}(p([a, b]), \delta)$ and $p([a, b]) \subset$ $\operatorname{Nbh}([p(a), p(b)], \delta)$.

By increasing $d$, we can assume that $d \gg \delta$ (increasing $d$ doesn't decrease $\epsilon$ ) where $\delta$ is the constant of Proposition 3 .

Let $\alpha$ be a geodesic in $T$, and define $\mathcal{C}(\alpha)$ to be the collection of vertex circles of $\alpha$. For any circle $C \subset S^{2} \subset \mathbb{R}^{3}$ the circumference of $C$ will be denoted $\operatorname{circ}(C)$.

Lemma 4 There is $S>0$ such that for any geodesic $\alpha$ in $T$,

$$
\sum_{C \in \mathcal{C}(\alpha)} \operatorname{circ}(C) \leq S[\operatorname{circ}(B)]
$$

where $B$ is the vertex of $\alpha$ closest to the vertices $\{\pi, v\}$ in $T$.

Proof If suffices to prove the following: There is a $\phi<1$, such that for any $B$, a vertex circle of $T$, and $C$, an adjacent vertex circle of $T$ with $B$ closer (in $T$ ) to $\{\pi, v\}$ than $C$, then $\operatorname{circ}(C) \leq \operatorname{circ}(B) \phi$. 
Using high school geometry, we can show that the Euclidean radius of a circle $E$ on $S^{2}$ is $e^{-h}$ where $h$ is the hyperbolic distance from $\mathbf{0}$ to the hyperbolic plane (Euclidian hemisphere in this model) whose boundary is $E$.

Let $S_{C}$ and $S_{B}$ be hyperbolic planes in $\mathbb{H}^{3}$ corresponding to $C$ and $B$ respectively. Let $\gamma$ be the shortest geodesic from 0 to $S_{C}$. Let $\beta$ be the shortest path in $\cup(\mathcal{P} \cup \mathcal{Y})$ with the same endpoints as $\gamma$. By Proposition 3, $\beta$ and $\gamma$ $\delta$-fellow travel. Notice that $\ell\left(\beta \cap S_{B}\right)>d$

It follows that $d\left(\mathbf{0}, S_{B}\right)+d-3 \delta \geq d\left(\mathbf{0}, S_{C}\right)$. Thus since $\frac{d}{2}<d-3 \delta$, letting $\phi=e^{-\frac{d}{2}}$ suffices. We have $\operatorname{circ}(C) \leq \operatorname{circ}(B) \phi$ since this is true for the radii.

Recall that is $X$ is the "tree" of the purple and yellow circles in the limit set of the Kleinian group $H, \Lambda(H) \subset S^{2}$. By Lemma 4, $X$ is a finite diameter geodesic metric space on which $H$ acts by homeomorphisms.

By orienting the intersection curve on $P / H_{P}$, we obtain a well defined orientation on the intersection line $P \cap Y$. Let $\pi$ and $v$ be the purple and yellow circles corresponding to $P$ and $Y$ respectively. We now denote $\pi \cap v=\{+,-\}$ where + is the point at infinity of the line $P \cap Y$ in orientation direction, and - is the other endpoint of $P \cap Y$. Since $H_{P}$ is a torsion free hyperbolic surface group, it follows that + is not a translate of - under the action of $H_{P}$, and similarly under the action of $H_{Y}$. However the stabilizer of $P$ in $H$ is $H_{P}$, and the stabilizer of $Y$ is $H_{Y}$, and so + is not a translate of - in $H$.

For any $\breve{\pi} \in \Pi$ and $\breve{v} \in \Upsilon$ which intersect there is $h \in H$ with $\breve{\pi}=h(\pi)$ and $\breve{v}=h(v)$. Thus $\breve{\pi} \cap \breve{v}=\{h(+), h(-)\}$. In short the intersection of any two circles in $X$ consists of a positive point $h(+)$ and a negative point $h(-)$.

Now consider the disjoint union $W=(\cup \Pi) \sqcup(\cup \Upsilon)$, that is $W$ is the disjoint union of the yellow and purple circles, so each path component of $W$ is a single circle where $W$ is given the path metric. Now for $\breve{\pi} \in \Pi$ and $\breve{v} \in \Upsilon$ where $\breve{\pi}$ and $\breve{v}$ intersect in $X$, define $+\breve{\pi}(\breve{v}) \in \breve{\pi} \subset W$ to be the positive intersection point in $\breve{\pi}$, and $-\breve{\pi}(\breve{v}) \in \breve{\pi} \subset W$ to be the negative intersection point in $\breve{\pi}$. Define $+_{\breve{v}}(\breve{\pi}),-\breve{v}(\breve{\pi}) \in \breve{v} \subset W$ similarly.

Notice that $H$ acts by homeomorphism on $W$ and that $X$ is the quotient space of $W$ by the equivalence relation $+\breve{v}(\breve{\pi})=+_{\breve{\pi}}(\breve{v})$ and $-\breve{v}(\breve{\pi})=-\breve{\pi}(\breve{v})$. Also the action of $H$ on $X$ is the quotient action of the action of $H$ on $W$.

We now form a different quotient of $W$ on which $H$ acts. Define the bassackward equivalence relation $\sim$ by $+_{\breve{v}}(\breve{\pi}) \sim-_{\breve{\pi}}(\breve{v})$ and $-_{\breve{v}}(\breve{\pi}) \sim+_{\breve{\pi}}(\breve{v})$. Notice 
that since + is not a translate of - , for any $h \in H \quad h$ preserves the equivalence classes of $W$. It follows that $H$ acts via homeomorphism on the quotient space $Z=W / \sim$. Where we give $Z$ the path metric calculating the arc length of a path in each circle.

Notice that the tree structure of $Z$ is exactly the same as the tree structure of $X$ (the same pairs of purple and yellow circles intersect), and so it follows from Lemma 4 that $Z$ is a finite diameter path metric space.

Now for any $h \in H$, consider $h: S^{2} \rightarrow S^{2}$. Since $h$ is conformal, then $h$ has continuous first derivative. Since $S^{2}$ is compact we have that $h: S^{2} \rightarrow S^{2}$ is Lipschitz with constant $c>0$. It follows that for any circle $C \subset S^{2}, h: C \rightarrow$ $h(C)$ is Lipschitz with constant $c$, even when $C$ and $h(C)$ are given the path metric inherited from $S^{2}$. Thus $h: Z \rightarrow Z$ is also Lipschitz with constant $c$. It follows that $h$ extends to a homeomorphism $h: \hat{Z} \rightarrow \hat{Z}$ where $\hat{Z}$ is the metric completion of $Z$.

Theorem $5 \quad \hat{Z}$ is compact.

Proof It suffices to show that $Z$ is totally bounded (for every $\epsilon>0$ there is a finite covering of $Z$ by $\epsilon$-balls). This follows easily from the compactness of a circle together with Lemma 4 , which implies that there are only a finite number of circles above a given size. Cover the finite collection of "large" circles with a finite collection of balls of very small radius. The collection of balls of radius $\epsilon$ with the same centers will cover $Z$.

Now the group $H$ acts by homeomorphism on the Peano continua without cut-points $\hat{Z}$. Let $\mathcal{A}=\Pi \cup \Upsilon$ (that is the elements of $\mathcal{A}$ are the circles). The collection of circles $\mathcal{A}$ is cross-connected, null and fine since this is true when $\mathcal{A}$ is consider as a collection of closed subset of $\Lambda(H)$, and there is a homeomorphism of $\hat{Z}$ to $\Lambda(H)$ which permutes the elements of $\mathcal{A}$. Thus $(H, \mathcal{A})$ is a fine pairing on $\hat{Z}$. Notice that for any circle $A \in \mathcal{A}$, the stabilizer $\operatorname{Stab}(A)$ is the same as it was in $\Lambda H$ (a coFuchsian subgroup) and the action of $\operatorname{Stab}(A)$ on $A$ is also the same (the action of a coFuchsian group on it limit circle). Thus for each $A \in \mathcal{A}, \operatorname{Stab}(A)$ acts on $A$ as convergence group. Now $(H, \mathcal{A})$ on $\hat{Z}$ satisfies all the hypothesis of the failed conjecture, but

Theorem 6 The action of $H$ on $\hat{Z}$ is not a convergence action.

Proof Recall that $\pi$ and $v$ are the circles corresponding to the planes $P$ and $Y$ resp. The stabilizer $\operatorname{Stab}(\pi)=H_{P}$ and $\operatorname{Stab}(v)=H_{Y}$. Recall that 
$H_{P} \cap H_{Y}=<g>$ where $g$ represents the intersection curve of $P / H_{P}$ and $Y / H_{Y}$. The sequence $\left(g^{i}\right)(i>0)$ acts as a convergence sequence on both $\pi$ and $v$. In its action on $\pi$, the attractor of $\left(g^{i}\right)$ is the point $+_{\pi}(v) \in \pi$, and the repeller is the point $-{ }_{\pi}(v) \in \pi$. On the other hand, in its action on $v,\left(g^{i}\right)$ has attractor $+_{v}(\pi) \in v$ and repeller $-{ }_{v}(\pi) \in v$. However in $\hat{Z},+{ }_{\pi}(v)=-{ }_{v}(\pi)$ and $+_{v}(\pi)=-{ }_{\pi}(v)$.

Let $n \in \hat{Z}$, and choose $a \in \pi-\left\{n,-{ }_{\pi}(v)\right\}$ and $b \in v-\left\{n,-{ }_{v}(\pi)\right\}$. We have that $g^{i}(a) \rightarrow+_{\pi}(v)$ and $g^{i}(b) \rightarrow-{ }_{v}(\pi)$, and the same would be true for subsequence of $\left(g^{i}\right)$. Thus $n$ is not the repeller of any convergence subsequence of $\left(g^{i}\right)$ (since there is not a single attractor), and so $\left(g^{i}\right)$ has no convergence subsequence and $H$ doesn't act as a convergence group on $\hat{Z}$.

\section{Bootstrapping}

Definition Let $X$ be a Peano continuum without cut points, and $G$ be a group which acts by homeomorphisms on $X$. Let $\mathcal{A}$ be a $G$-invariant collection of closed subsets of $X$ such that the following conditions are satisfied:

(1) $|A|>2$ for all $A \in \mathcal{A}$.

(2) $\mathcal{A}$ is null. That is: For any $\epsilon>0$, the set of elements of $\mathcal{A}$ with diameter at least $\epsilon,\{A \in \mathcal{A}: \operatorname{diam}(A)>\epsilon\}$ is finite.

(3) $\mathcal{A}$ is fine. That is: For any $x, y \in X$ there exists a finite $\mathcal{B} \subset \mathcal{A}$ such that $\cup \mathcal{B}$ separates $x$ from $y$.

(4) For each $A \in \mathcal{A}$, the stabilizer of $A$, $\operatorname{Stab}(A)=\{g \in G: g(A)=A\}$ acts as a convergence group on $A$.

(5) For any $A, B \in \mathcal{A}$ with $|A \cap B| \leq 2$ :

- If $A \cap B=\{c\}$, then for any $b \in B-\{c\}$ there exists finite crossing sequence $A, A_{1}, \ldots A_{n}, B \in \mathcal{A}$ with $D \cap A_{i}=\emptyset$ for all $1 \leq i \leq n$, where $D=\{b, c\}$.

- If $|A \cap B| \neq 1$ then there is a crossing sequence $A, A_{1}, \ldots A_{n}, B \in \mathcal{A}$ with $D \cap A_{i}=\emptyset$ for all $1 \leq i \leq n$, where $D=A \cap B$.

If these conditions are satisfied then we say that the pair $(G, \mathcal{A})$ is a local convergence pairing on $X$. Clearly every local convergence pairing is a fine pairing. 
We will show that if $(G, \mathcal{A})$ is a local convergence pairing on $X$, then $G$ acts as a convergence group on $X$. We translate the following two results from [13], the second of which is not due to the author, into the setting we are interested in.

Lemma 7 Let $(G, \mathcal{A})$ be a local convergence pairing on $X$ and $\mathcal{B} \subset \mathcal{A}$ with $\mathcal{B}$ finite and cross-connected. If $\left(g_{i}\right)$ is a sequence of elements of $G$ then either $\left(g_{i}(\cup \mathcal{B})\right)$ is null or there exists $B \in \mathcal{B}, A \in \mathcal{A}$ and a subsequence $\left(g_{i_{n}}\right)$ of $\left(g_{i}\right)$ with $g_{i_{n}}(B)=A$ for all $n$.

Lemma 8 If $M$ is a compact connected locally connected Hausdorff space and a closed set $A$ doesn't separate the nonempty connected set $B \subset M-A$ from the nonempty connected set $C \subset M-A$, then there exists a neighborhood $V$ of $A$ which doesn't separate $B$ from $C$.

Lemma 9 Let $Y$ be a Peano continuum without cut-points. For any point $x \in Y$ and for any $\epsilon>0$ there is a $\delta>0$ such that $Y-B(x, \epsilon)$ is contained in a single component of $Y-B(x, \delta)$.

Proof Suppose not, then there exists $\epsilon>0$, such that for any $\delta>0, Y-$ $B(x, \epsilon)$ is not contained in a single component of $Y-B(x, \delta)$. In particular, for each $n>\frac{1}{\epsilon}$, there exist $a_{n}, b_{n} \in Y-B(x, \epsilon)$ such that $a_{n}$ and $b_{n}$ lie in different components of $Y-B\left(x, \frac{1}{n}\right)$. By compactness, we may assume that $a_{n} \rightarrow a \in Y-B(x, \epsilon)$ and $b_{n} \rightarrow b \in Y-B(x, \epsilon)$. Let $A$ and $B$ be closed connect neighborhoods of $a$ and $b$ respectively with $x \notin A \cup B$. There exists a $\delta>0$ such that $A \cap B(x, \delta)=\emptyset=B \cap B(x, \delta)$ and (using Lemma 8 ) $B(x, \delta)$ does separate $A$ from $B$. This contradicts the choice of $x_{n}$ and $y_{n}$ for $n \gg 0$.

Lemma 10 Let $(G, \mathcal{A})$ be a local convergence pairing on $X$, and suppose $B, A_{1}, \ldots A_{m} \in \mathcal{A}$ is a crossing sequence. Let $\left(f_{i}\right) \subset G$ act as a convergence sequence on $B$ with attractor $p$ and repeller $n$. If for all $1 \leq j \leq m$, we have that $n, f_{i}^{-1}(p) \notin A_{j}$ for all $i$, then $f_{i}\left(A_{j}\right) \rightarrow p$ for all $1 \leq j \leq m$.

Proof By nullity, since $p \notin f_{i}\left(A_{j}\right)$, it suffices to show that $d\left(p, f_{i}\left(A_{j}\right)\right) \rightarrow 0$.

Proceeding by induction consider the case where $j=1$. If $B \cap A_{1} \neq \emptyset$, then there is $a \in\left(B \cap A_{1}\right)-\{n\}$ and $f_{i}(a) \rightarrow p$, yielding $d\left(p, f_{i}\left(A_{1}\right)\right) \rightarrow 0$. Now consider the case where $B$ separates $A_{1}$ and $A_{1}$ separates $B$. Let $B^{+}$be the intersection of $B$ with that component of $X-A_{1}$ which contains $p$, and let $B^{-}=B-B^{+}$. We know from [15] that $n$ is not an isolated point of $B$, so 
$B^{ \pm} \neq\{n\}$. Let $b \in B^{-}-\{n\}$ and $\hat{b} \in B^{+}-\{n\}$. We have that $f_{i}(b), f_{i}(\hat{b}) \rightarrow p$, but $f_{i}\left(A_{1}\right)$ separates $f_{i}(b)$ from $f_{i}(\hat{b})$. It follows from local connectivity of $X$ that $d\left(p, f_{i}\left(A_{1}\right)\right) \rightarrow 0$.

Given that $f_{i}\left(A_{j}\right) \rightarrow p$, one of the following is true:

- $\exists a \in A_{j} \cap A_{j+1}$ and so $f_{i}(a) \rightarrow p$.

- $f_{i}\left(A_{j}\right)$ separates $f_{i}\left(A_{j+1}\right)$ and by Lemma $9 d\left(p, f_{i}\left(A_{j+1}\right)\right) \rightarrow 0$.

Either way $d\left(p, f_{i}\left(A_{j+1}\right)\right) \rightarrow 0$ and so $f_{i}\left(A_{j+1}\right) \rightarrow p$.

Lemma 11 Let $(G, \mathcal{A})$ be a local convergence pairing on $X$, and $A, B \in \mathcal{A}$. If $\left(f_{i}\right) \subset \operatorname{Stab}(A) \cap \operatorname{Stab}(B)$ acts as a convergence sequence on both $A$ and $B$ with $p$ the attractor of $\left(f_{i}\right)$ in its action on $A$ and on $B$ then the repeller $n$ of $\left(f_{i}\right)$ in its action on $A$ is also the repeller of $\left(f_{i}\right)$ in its action of $B$.

Proof If $A \cap B \neq\{p\}$, then there is $a \in A \cap B-\{p\}$ and the convergence sequence $\left(f_{i}^{-1}\right)$ has attractor $n$ in its action on $A$ and so $f_{i}^{-1}(a) \rightarrow n$. Thus the convergence sequence $\left(f_{i}^{-1}\right)$ has attractor $n$ in its action on $B$. It follows that the repeller of $\left(f_{i}\right)$ in its action on $B$ is $n$.

Now we deal with the case where $A \cap B=\{p\}$. Suppose that the repeller of $\left(f_{i}\right)$ in its action on $B$ is $\hat{n} \neq n$. Notice that $f_{i}(p)=p$ for all $i$.

By (5) there is a finite crossing sequence $A, A_{1}, \ldots A_{m}, B$ with $p \notin A_{j}$ for all $j$, and also passing to a subsequence of $\left(f_{i}\right)$, we may assume that $f_{i}(n) \notin A_{j}$ for all $i$ and $1 \leq j \leq m$.

We now apply Lemma 10 to $\left(f_{i}^{-1}\right.$ ) ( which has attractor $n$ and repeller $p$ in its action on $A$ ) and $A, A_{1}, \ldots A_{m}$ we find that $f_{i}^{-1}\left(A_{m}\right) \rightarrow n$. Applying Lemma 10 to $\left(f_{i}^{-1}\right)$ and the sequence $B, A_{m}$ we get that $f_{i}^{-1}\left(A_{m}\right) \rightarrow \hat{n}$. Thus $n=\hat{n}$.

Theorem 12 Let $(G, \mathcal{A})$ be a local convergence pairing on $X$, and $A, B \in \mathcal{A}$ with $A \cap B \neq \emptyset$. If $\left(f_{i}\right) \subset G$ acts as a convergence sequence on both $A$ and $B$ and $n$ is the repeller and $p$ the attractor of $\left(f_{i}\right)$ in its action on $A$, then the attractor of $\left(f_{i}\right)$ in its action on $B$ is $p$ and the repeller of $\left(f_{i}\right)$ in its action on $B$ is $n$.

Proof Suppose we have distinct $a, b \in A \cap B-\{n\}$, with $f_{i}(a) \rightarrow p$ and $f_{i}(b) \rightarrow p$. It follows that $p$ is the attractor of $\left(f_{i}\right)$ in its action on $B$, and so by Lemma $11 n$ is the repeller of $\left(f_{i}\right)$ in its action on $B$. 
Similarly if we have distinct $a, b \in A \cap B-\{p\}$, then $\left(f_{i}^{-1}\right)$ has attractor $n$ and repeller $p$ in its action on $B$, and so $p$ is the attractor, and $n$ the repeller of $\left(f_{i}\right)$ in its action on $B$.

We are left with the case where $A \cap B \subset\{n, p\}$. Replacing $\left(f_{i}\right)$ with $\left(f_{i}^{-1}\right)$ if need be (and so exchanging the rolls of $n$ and $p$ ), we may assume that $n \in A \cap B$.

Case I $p \in A \cap B$

Notice that $\left(f_{i}\right)$ leaves $A \cap B$ invariant. By (5) there is a crossing sequence $A, A_{1}, \ldots A_{m}, B$ with $p, n \notin A_{j}$ for all $j$. Since $f_{i}^{-1}(p) \notin A_{j}$ for all $i$ and $j$, Lemma 10 implies that $f_{i}\left(A_{m}\right) \rightarrow p$.

If $A_{m} \cap B=\emptyset$, then by Lemma 10 applied to $\left(f_{i}\right)$ and the crossing sequence $B, A_{m}, f_{i}\left(A_{m}\right)$ must converge to the attractor of $\left(f_{i}\right)$ in its action on $B$, but $f_{i}\left(A_{m}\right) \rightarrow p$, so $p$ is the attractor of $\left(f_{i}\right)$ in its action on $B$ and Lemma 11] implies that $n$ is the repeller of $\left(f_{i}\right)$ in its action on $B$.

If not there exists $b \in A_{m} \cap B$. It follows that $f_{i}(b) \rightarrow p$. Since $\left(f_{i}\right)$ leaves that set $A \cap B$ invariant it follows from 14 that the attractor and repeller of $\left(f_{i}\right)$ in its action on $B$ are contained in the set $A \cap B$. All together this implies that $p$ is the attractor of $\left(f_{i}\right)$ in its action on $B$ and by Lemma 11 that $n$ is the repeller of $\left(f_{i}\right)$ in its action on $B$.

Case II $p \notin A \cap B$

By (5D) there is a crossing sequence $A, A_{1}, \ldots A_{m}, B$ with $n \notin A_{j}$ for all $j$, and also passing to a subsequence of $\left(f_{i}\right)$, we may assume that $f_{i}^{-1}(p) \notin A_{j}$ for all $i$ and $j$. Thus we may apply Lemma 10 to show that $f_{i}\left(A_{m}\right) \rightarrow p$.

If $A_{m} \cap B \neq \emptyset$, then $f_{i}\left(A_{m} \cap B\right) \rightarrow p$, so $p \in B$ which puts us back in Case I.

If $A_{m} \cap B=\emptyset$, then by Lemma 10 applied to $\left(f_{i}\right)$ and the crossing sequence $B, A_{m}$, we find that $f_{i}\left(A_{m}\right)$ converges to the attractor of $\left(f_{i}\right)$ in its action on $B$. It follows that $p$ is the attractor of $\left(f_{i}\right)$ in its action on $B$, so $p \in B$ which puts us back in Case I.

Lemma 13 Let $(G, \mathcal{A})$ be a local convergence pairing on $X$ and $A, B \in \mathcal{A}$. If $\left(g_{i}\right) \subset \operatorname{Stab}(A)$ acts as a convergence sequence on $A$ with attractor $p$ and repeller $n$, and $A \cap B \neq \emptyset$, then there exists a subsequence $\left(h_{i}\right) \subset\left(g_{i}\right)$ such that for any compact $C \subset B-\{n\}, h_{i}(C) \rightarrow p$.

Proof The proof has two cases. 
Case I $\operatorname{diam}\left(g_{i}(B)\right) \not \rightarrow 0$

By Lemma 7 there is $D \in A$ and a subsequence $\left(k_{i}\right) \subset\left(g_{i}\right)$ with $k_{i}(B)=D$ for all $i$. Let $f_{i}=k_{1}^{-1} k_{i} \in \operatorname{Stab}(A) \cap \operatorname{Stab}(B)$. Clearly $\left(f_{i}\right)$ acts as a convergence sequence on $A$ with attractor $k_{1}^{-1}(p)$ and repeller $n$. Using (4) we find a subsequence $\left(q_{i}\right) \subset\left(f_{i}\right)$ with $\left(q_{i}\right)$ acting as a convergence subsequence on $B$. By Theorem $12\left(q_{i}\right)$ has attractor $k_{1}^{-1}(p)$ and repeller $n$ in its action on $B$. Let $h_{i}=k_{1} q_{i}$ so $\left(h_{i}\right) \subset\left(k_{i}\right) \subset\left(g_{i}\right)$. For any compact $C \subset B-\{n\}$, by definition $q_{i}(C) \rightarrow k_{1}^{-1}(p)$. It follows that $h_{i}(C) \rightarrow p$.

Case II $\operatorname{diam}\left(g_{i}(B)\right) \rightarrow 0$

If there is $a \in A \cap B-\{n\}$ then $g_{i}(a) \rightarrow p$ and so $g_{i}(B) \rightarrow p$ and we are done. Thus we need only consider the case where $A \cap B=\{n\}$. Using (15) and passing to a subsequence $\left(h_{i}\right) \subset\left(g_{i}\right)$ we obtain a crossing sequence $A, A_{1}, \ldots A_{m}, B$ with $n \notin A_{j}$ for all $j$, and $h_{i}^{-1}(p) \notin A_{j}$ for all $i$ and $j$. Thus by Lemma 10, $h_{i}\left(A_{m}\right) \rightarrow p$.

If $b \in A_{m} \cap B$ we get that $h_{i}(b) \rightarrow p$ and so $h_{i}(B) \rightarrow p$.

If $A_{m} \cap B=\emptyset$, then for each $i, h_{i}(B)$ is separated by the set $h_{i}\left(A_{m}\right)$. It follows from Lemma 9 that $d\left(p, h_{i}(B)\right) \rightarrow 0$ and so $h_{i}(B) \rightarrow p$.

Corollary 14 Let $(G, \mathcal{A})$ be a local convergence pairing on $X$ and $A, B \in \mathcal{A}$. If $\left(g_{i}\right) \subset \operatorname{Stab}(A)$ acts as a convergence sequence on $A$ with attractor $p$ and repeller $n$, then there exists a subsequence $\left(h_{i}\right) \subset\left(g_{i}\right)$ such that for any compact $C \subset B-\{n\}, h_{i}(C) \rightarrow p$.

Proof Using (5) we find a minimal crossing sequence $A, A_{1} \ldots A_{m-1}, A_{m}=$ $B \in \mathcal{A}$. If $A_{1} \cap A=\emptyset$ then by Lemma 10, $g_{i}\left(A_{1}\right) \rightarrow p$. If $A_{1} \cap A \neq \emptyset$ we apply Lemma 13 and pass to a subsequence of $\left(g_{i}\right)$ so that for any compact $C \subset A_{1}-\{n\}, g_{i}(C) \rightarrow p$.

Now by induction we may assume that for any compact $C \subset A_{k}-\{n\}, g_{i}(C) \rightarrow$ $p$. If $a \in A_{k+1} \cap A_{k}$, then since $A_{k+1} \cap A=\emptyset, g_{i}(a) \rightarrow p$ and it follows that $g_{i}\left(A_{k+1}\right) \rightarrow p$.

Now consider when $A_{k+1} \cap A_{k}=\emptyset$. Either $g_{i}\left(A_{k}\right) \rightarrow p$ and by Lemma 9 $g_{i}\left(A_{k+1}\right) \rightarrow p$.

Otherwise diam $g_{i}\left(A_{k}\right) \not \rightarrow 0$, and arguing as in Case I of Lemma 13 we obtain a convergence sequence acting on $A_{k}$, and applying Lemma 10 to this convergence sequence and the crossing sequence $A_{k}, A_{k+1}$ gives us a subsequence $\left(h_{i}\right) \subset\left(g_{i}\right)$ with $h_{i}\left(A_{k+1}\right) \rightarrow p$. 
Theorem 15 If $(G, \mathcal{A})$ is a local convergence pairing on $X$ then $G$ acts as a convergence group on $X$.

Proof By Theorem 1 it suffices to show that for each $A \in \mathcal{A}, \operatorname{Stab}(A)$ acts as a convergence group on $X$.

Suppose not, then there is $\left(f_{i}\right) \subset \operatorname{Stab}(A)$ with $\left(f_{i}\right)$ acting as a convergence sequence on $A$ with attractor $p$ and repeller $n$, but no subsequence of $\left(f_{i}\right)$ acts as convergence sequence on $X$ with attractor $p$ and repeller $n$. Using the compactness of $X$, and passing to a subsequence, we may assume there is a sequence $\left(x_{i}\right) \subset X-\{n\}$ with $x_{i} \rightarrow x \neq n$ and $f_{i}\left(x_{i}\right) \rightarrow \hat{x} \neq p$. Choose a finite $\mathcal{B} \subset \mathcal{A}$ such that $\cup \mathcal{B}$ separates $n$ from $x$. Using (5) and Corollary 14] passing to subsequences we may assume that $f_{i}(B) \rightarrow p$ for all $B \in \mathcal{B}$, so $f_{i}(\cup \mathcal{B}) \rightarrow p$.

Let $U$ be the component of $X-\cup \mathcal{B}$ which contains $n$. Thus $\partial U \subset \cup \mathcal{B}$ and so $f_{i}(\partial U) \rightarrow p$. Notice that $f_{i}(A-U) \rightarrow p$ so $\operatorname{diam}\left(f_{i}(A-U)\right) \rightarrow 0$ and this implies that $\operatorname{diam} f_{i}(U \cap A)$ is bounded away from 0 (since $|A|>2$ ), and so $\operatorname{diam}\left(g_{i}(U)\right)$ is bounded away from 0 . Since $p$ is not a cut point one can show using Lemma 8 that $f_{i}(X-U) \rightarrow p$, but for all $i \gg 0 x_{i} \in X-U$. Thus $f_{i}\left(x_{i}\right) \rightarrow p$ contradicting $\hat{x} \neq p$.

Lemma 16 Let $Z$ be a Peano continuum which admits no essential map to the circle, and let $A, B$ be disjoint closed subsets of $Z$. Let $c, d \in Z-[A \cup B]$. If $c$ and $d$ are not separated by $A$ nor separated by $B$, then $c$ and $d$ are not separated by $A \cup B$.

Janiszewski proved this in the case where $Z$ was the 2-sphere 9, XVII 5.2].

Proof Suppose not, that is $c$ and $d$ lie in different components of $X-[A \cup B]$. Let $S^{1}=I \cup J$ where $I$ and $J$ are arcs with $I \cap J=\{\hat{c}, \hat{d}\}$. Let $\hat{a} \in \operatorname{Int} I$ and $\hat{b} \in \operatorname{Int} J$. Let $\hat{U}$ be the arc of $S^{1}$ from $\hat{a}$ to $\hat{b}$ passing through $\hat{c}$, and similarly defined $\hat{V}$ to be the arc of $S^{1}$ from $\hat{a}$ to $\hat{b}$ passing through $\hat{d}$.

The space $X$ is locally path connected since it is a Peano continuum and so we may choose a mapping $\alpha: I \rightarrow X$ with $\alpha(\hat{c})=c$, and $\alpha(\hat{d})=d$ with the property that $\alpha(I) \cap B=\emptyset$ (since $B$ doesn't separated). Similarly we choose a map $\beta: J \rightarrow X$ with $\beta(\hat{c})=c$, and $\beta(\hat{d})=d$ with the property that $\beta(J) \cap A=\emptyset$. We paste $\alpha$ and $\beta$ to obtain $\gamma: S^{1} \rightarrow X$.

Let $X-[A \cup B]=U \cup V$ where $U$ and $V$ are open sets of $X, U \cap V=\emptyset$ and $c \in U$ and $d \in V$. By the Tietze Extension theorem, there exists a map $f: U \cup A \cup B \rightarrow \hat{U}$ with $f(A)=\{\hat{a}\}$ and $f(B)=\{\hat{b}\}$. Similarly there exists a 
map $g: V \cup A \cup B \rightarrow \hat{V}$ with $g(A)=\{\hat{a}\}$ and $g(B)=\{\hat{b}\}$. We paste $f$ and $g$ to get a continuous $h: X \rightarrow S^{1}$. By construction $h \circ \gamma: S^{1} \rightarrow S^{1}$ is homotopic to the identity map and so essential. Clearly $h$ is essential since $h \circ \gamma$ is essential. Thus $X$ admits an essential map, $h$, to $S^{1}$ which is a contradiction.

Corollary 17 Let $Z$ be a Peano continuum which admits no essential map to the circle, let $A$ be a closed subset of $Z$, and let $c, d \in Z-A$. If $c$ and $d$ are separated by $A$, then $c$ and $d$ are separated by some component of $A$.

Proof Let $\left(A_{i}\right)$ be a nested sequence $\left(A_{i+1} \subseteq A_{i}\right)$ of closed subsets of $A$ with the property that for each $i, A_{i}$ separates $c$ from $d$. By Lemma $8 \bigcap_{i} A_{i}$ separates $c$ from $d$. Thus by Zorn's Lemma, there exists a minimal closed subset $B$ of $A$ which separates $c$ from $d$. Since no proper closed subset of $B$ separates $c$ from $d$, by Lemma 16, $B$ is connected.

Main Theorem Let $X$ be a Peano continuum, without cut points, which does not admit an essential map to the circle. If $G$ acts on $X$ by homeomorphisms and $\mathcal{A}$ is a $G$-invariant collection of connected closed subsets of $X$, then $G$ acts as a convergence group on $X$ provided the following conditions are satisfied:

- $\mathcal{A}$ is null, that is for any $\epsilon>0$, the set of elements of $\mathcal{A}$ with diameter at least $\epsilon,\{A \in \mathcal{A}: \operatorname{diam}(A)>\epsilon\}$, is finite.

- $\mathcal{A}$ is fine, that is for any $x, y \in X$ there exists a finite $\mathcal{B} \subset \mathcal{A}$ such that $\cup \mathcal{B}$ separates $x$ from $y$.

- $\operatorname{Stab}(A)$ acts as a convergence group on $A$ for each $A \in \mathcal{A}$.

Proof First we remove from $\mathcal{A}$ all singleton sets (sets with only one element). We must verify that this new $\mathcal{A}$ satisfies the three original hypothesis. Two of them are obvious, and the fact that $\mathcal{A}$ is still fine follows from $X$ having no cut points and Corollary [17. Thus we may assume that $\mathcal{A}$ has no singletons, and since each element of $\mathcal{A}$ is connected, each element of $\mathcal{A}$ is uncountable.

We must show that $(G, \mathcal{A})$ is a local convergence pairing on $X$. We are given all of the conditions for a local convergence pairing except (5) which we state again here. For any $A, B \in \mathcal{A}$ with $|A \cap B| \leq 2$ :

- If $A \cap B=\{c\}$ we choose any $b \in B-\{c\}$ and let $D=\{b, c\}$.

- If $|A \cap B| \neq 1$ we let $D=A \cap B$.

there exists finite crossing sequence $A_{1}, A_{2}, \ldots A_{n} \in \mathcal{A}$ with $A_{1} \cap A \neq \emptyset$, and $A_{n} \cap B \neq \emptyset$ but $D \cap A_{i}=\emptyset$ for all $1 \leq i \leq n$.

There are three cases. 
Case I $\quad A \cap B=\emptyset$

We must find a crossing sequence in $\mathcal{A}$ from $A$ to $B$.

We start by showing that $\cup \mathcal{A}$ is connected. Suppose not, then there are open sets $U, V$ of $X$ with $\cup \mathcal{A} \subset U \cup V, \cup \mathcal{A} \cap U \cap V=\emptyset, \cup \mathcal{A} \cap U \neq \emptyset \neq \cup \mathcal{A} \cap V$. By Corollary 17. we may assume that $\partial U$ is connected. Since $X$ has no cut points, there exist distinct $x, y \in \partial U$. By fine, there exist a finite $\mathcal{F} \subset \mathcal{A}$ such that $\cup \mathcal{F}$ separates $x$ and $y$. Since $\cup \mathcal{F}$ separates $x$ from $y$, and $\partial U$ is connected, then $\cup \mathcal{F} \cap \partial U \neq \emptyset$. This is a contradiction since $\cup \mathcal{F} \subset U \cup V$. Thus $\cup \mathcal{A}$ is connected.

We now show that $\cup \mathcal{A}$ is cross connected. We define the cross-component of $E \in \mathcal{A}$ as

$$
I(E)=\cup\{C \in \mathcal{A} \text { : there is a crossing sequence in } \mathcal{A} \text { from } E \text { to } C\}
$$

If $e \in E$, we define $I(e)=\beth(E)$ (note that this is well defined).

Claim For each $e \in \cup \mathcal{A}$, there exists a basis $\left\{U_{\alpha}\right\}$ of connected open sets at $e$ in $X$ with the property that $\partial U_{\alpha} \subset I(e)$ for all $\alpha$.

Let $z \in X-\{e\}$ and $y \in I(e)-\{e, z\}$. By fineness, there is a finite $\mathcal{F} \subset \mathcal{A}$ so that the component $U$ of $X-\cup \mathcal{F}$ which contains $e$ has the property that $y, z \notin \bar{U}$. Since $e$ is not a cut point, using compactness and Lemma 8 we may assume that $\bar{U}$ doesn't separate $y$ from $z$. Thus $\partial U$ separates $e$ from $\{y, z\}$ which is contained in a single component of $X-\partial U$. By Corollary 17 there is a single component $P$ of $\partial U$ which separates $e$ from $y$ (of course $P$ also separates $e$ from $z$ ). Since $e, y \in I(e)$, which is connected, $P \cap I(e) \neq \emptyset$. It follows that $P \subset J(e)$ since $P$ is a connected subset of a finite union of elements of $\mathcal{A}$. Thus we have show that for every $z \in X-\{e\}$ there is a connected open set $V \ni e$ with $\partial V \subset \exists(e)$ but $z \notin \bar{V}$. Using compactness, the Claim follows.

Let $a \in A$ and $b \in B$, and choose $U_{a}, U_{b}$, disjoint connected open neighborhoods of $a$ and $b$ respectively, with $\partial U_{a} \subset J(a)$ and $\partial U_{b} \subset \exists(b)$. Now for every other $e \in \cup \mathcal{A}$ choose $U_{e}$, connect open neighborhood of $e$, with the property that $\partial U_{e} \subset \mathcal{I}(e)$ and $a, b \notin U_{e}$. The collection $\left\{U_{e}: e \in \cup \mathcal{A}\right\}$ is an open cover of $\cup \mathcal{A}$ Since $\cup \mathcal{A}$ is connected there exists a finite chain of open sets $U_{e_{1}}, \ldots U_{e_{n}}$, with $U_{e_{i}} \cap U_{e_{i+1}} \neq \emptyset$ for each $1 \leq i \leq n$ where $a=e_{1}$ and $b=e_{n}$. Notice that since $U_{a} \cap U_{b}=\emptyset, n>2$. We may assume this chain is minimal.

We now show that for any $1<i<n, \boldsymbol{I}\left(e_{i-1}\right)=\mathbf{I}\left(e_{i}\right)=\mathbf{I}\left(e_{i+1}\right)$. Since the chain is minimal, $U_{e_{i-1}} \cap U_{e_{i+1}}=\emptyset, U_{e_{i-1}}-\overline{U_{e_{i}}} \neq \emptyset$ and $U_{e_{i+1}}-\overline{U_{e_{i}}} \neq \emptyset$. Thus $U_{e_{i-1}}-\partial U_{e_{i}}$ is not connected and and neither is $U_{e_{i-1}}-\partial U_{e_{i}}$. Since $U_{e_{i-1}}$ and 
$U_{e_{i+1}}$ are connected sets, it follows that $\partial U_{e_{i}} \cap U_{e_{i-1}} \neq \emptyset \neq \partial U_{e_{i}} \cap U_{e_{i+1}}$. Notice that $\partial U_{e_{i}} \subset \exists\left(e_{i}\right)$ which is connected. Since $U_{e_{i-1}} \cap U_{e_{i+1}}=\emptyset, J\left(e_{i}\right) \cap \partial U_{e_{i-1}} \neq$

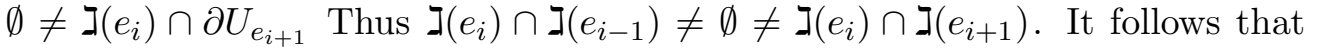
$\beth\left(e_{i-1}\right)=\beth\left(e_{i}\right)=\Xi\left(e_{i+1}\right)$.

Thus by induction, $\Xi(A)=\Xi(a)=\Xi(b)=\Xi(B)$ and so there is a finite crossing sequence from $A$ to $B$ as required.

Case II $|A \cap B|=\{c\}$

Choose some $b \in B-\{c\}$ and some $a \in A-\{c\}$. Using compactness of $X$

and fineness it can be shown that for any neighborhood $W$ of $c$, there exists a neighborhood $U \subset W$ of $c$ and a finite subset $\mathcal{F} \subset \mathcal{A}$ such that $\partial U \subset \mathcal{F}$. Using Lemma 8 can find an open neighborhood $U$ of $c$ with $a, b$ in the same component $N$ of $X-U$ and with $\partial U \subset \cup \mathcal{F}$ where $\mathcal{F}$ is a finite subset of $\mathcal{A}$. Using the nullness of $\mathcal{A}$, we may assume that $a, b \notin \cup \mathcal{F}$. By Lemma 16] there is a component $\cup \mathcal{E}$ of $\cup \mathcal{F}$ which separates $c$ from $N$. Thus $\mathcal{E}$ is cross-connected and $\{b, c\} \cap \cup \mathcal{E}=\emptyset$. Since $\cup \mathcal{E}$ separates $a$ from $c$ and separates $b$ from $c$, there is a crossing sequence in $\mathcal{E}$ from $A$ to $B$ missing the set $\{b, c\}$.

Case III $|A \cap B|=2$

This is similar to (but easier than) the previous case and will be left to the reader (simply find a finite subset of $\mathcal{A}$ which separates the two points of intersection).

\section{References}

[1] Mladen Bestvina, Geoffrey Mess, The boundary of negatively curved groups, J. Amer. Math. Soc. 4 (1991) 469-481 MathReview

[2] B H Bowditch, A topological characterisation of hyperbolic groups, J. Amer. Math. Soc. 11 (1998) 643-667 MathReview

[3] B H Bowditch, Peripheral splittings of groups, Trans. Amer. Math. Soc. 353 (2001) 4057-4082 (electronic) MathReview

[4] B H Bowditch, Boundaries of geometrically finite groups, Math. Z. 230 (1999) 509-527 MathReview

[5] B H Bowditch, Connectedness properties of limit sets, Trans. Amer. Math. Soc. 351 (1999) 3673-3686 MathReview

[6] James W Cannon, The combinatorial structure of cocompact discrete hyperbolic groups, Geom. Dedicata 16 (1984) 123-148 MathReview 
[7] James W Cannon, The theory of negatively curved spaces and groups, from: "Ergodic theory, symbolic dynamics, and hyperbolic spaces (Trieste, 1989)", Oxford Univ. Press (1991) 315-369 MathReview

[8] Andrew Casson, Douglas Jungreis, Convergence groups and Seifert fibered 3-manifolds, Invent. Math. 118 (1994) 441-456 MathReview

[9] James Dugundji, Topology, Allyn and Bacon, Inc. Boston, Mass. (1966) MathReview

[10] David Gabai, Convergence groups are Fuchsian groups, Ann. of Math. (2) 136 (1992) 447-510 MathReview

[11] M Gromov, Hyperbolic groups, from: "Essays in group theory", Math. Sci. Res. Inst. Publ. 8, Springer, New York (1987) 75-263 MathReview

[12] E L Swenson, Axial pairs and convergence groups on $S^{1}$, Topology 39 (2000) 229-237 MathReview

[13] Eric L Swenson, Convergence groups from subgroups, Geom. Topol. 6 (2002) 649-655 (electronic) MathReview

[14] Pekka Tukia, Homeomorphic conjugates of Fuchsian groups, J. Reine Angew. Math. 391 (1988) 1-54 MathReview

[15] Pekka Tukia, Convergence groups and Gromov's metric hyperbolic spaces, New Zealand J. Math. 23 (1994) 157-187 MathReview

[16] Asli Yaman, A topological characterisation of relatively hyperbolic groups, J. Reine Angew. Math. 566 (2004) 41-89 MathReview

Mathematics Department, Brigham Young University

Provo, UT 84604, USA

Email: eric@math.byu.edu

Received: 16 June 2004

Algebraic 83 Geometric Topology, Volume 5 (2005) 